
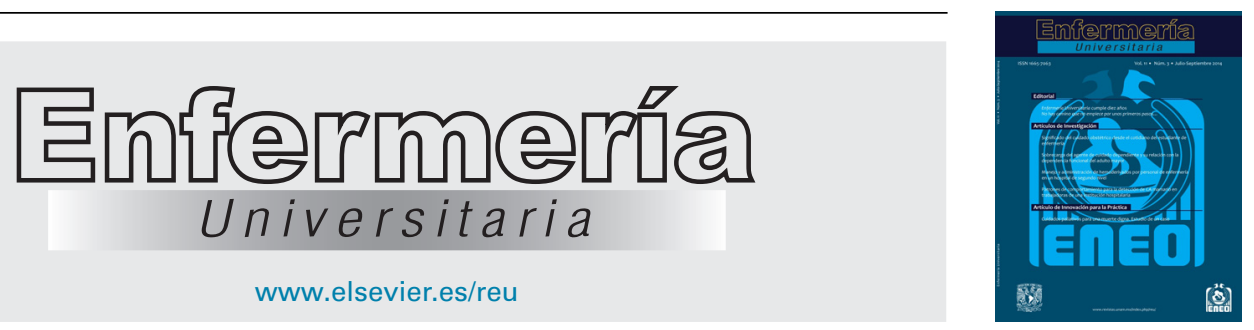

ARTÍCULO DE INVESTIGACIÓN

\title{
Cura húmeda de úlceras por presión. Atención en el ámbito domiciliar
}

\author{
I. Ortiz-Vargas ${ }^{a, *}$, M.L. García-Campos ${ }^{b}$, V. Beltrán-Campos ${ }^{b}$, F. Gallardo-López ${ }^{c}$, \\ A. Sánchez-Espinosa ${ }^{a}$ y M.E. Ruiz Montalvo ${ }^{a}$
}

a Facultad de Enfermería, Universidad Veracruzana, Región Veracruz, Veracruz, México

b Departamento de Enfermería Clínica, División de Ciencias de Salud e Ingenierías del Campus Celaya-Salvatierra, Universidad de Guanajuato, Guanajuato, México

c Departamento de Investigación del Colegio de Postgraduados, Campus Veracruz, Veracruz, México

Recibido el 29 de octubre de 2016; aceptado el 28 de junio de 2017

Disponible en Internet el 10 de noviembre de 2017

\author{
PALABRAS CLAVE \\ Úlceras por presión; \\ Cuidados de \\ enfermería en el \\ hogar; \\ Cicatrización \\ de heridas; \\ Apósitos \\ hidrocoloidales; \\ México
}

\begin{abstract}
Resumen La necesidad de trabajar en el domicilio el tratamiento y prevención de las úlceras por presión (UPP) mediante sesiones basadas en cura húmeda es trascendente para la eversión y cicatrización en menor tiempo.

Objetivo: Evaluar la eficacia del tratamiento de la cura húmeda en pacientes con UPP en un ambiente domiciliario.

Método: Se realizó una intervención clínica con la técnica de cura húmeda en 11 UPP de 4 pacientes egresados del hospital con su problema de salud resuelto, pero no así las UPP observadas en puntos de presión, con lesiones grado $\|(n=6)$ y grado III $(n=5)$. Se realizaron 12 curaciones, cada $72 \mathrm{~h}$, en los domicilios de las personas. Se midió la severidad y su reversión con el instrumento Pressure Ulcers Scale for Healing (PUSH). Este instrumento tiene un alfa de Cronbach de 0.823 .

Resultados: Se curaron 9 UPP de 11; todas las de grado ॥ $(n=6)$, mientras que de las de grado II se curaron $3(n=5)$. La cicatrización se dio en un lapso de 4 semanas, inferior a la cura tradicional que demora 2 veces más el tiempo de cicatrización.

Conclusión: En el ámbito domiciliario, la cura húmeda fue eficaz para revertir las UPP de pacientes que las habían desarrollado durante su hospitalización y que egresaron sin tratamiento para ellas. La intervención se convierte en una opción para mejorar la calidad de vida de las personas y un medio que las instituciones de salud pueden poner en práctica.

(c) 2017 Universidad Nacional Autónoma de México, Escuela Nacional de Enfermería y Obstetricia. Este es un artículo Open Access bajo la licencia CC BY-NC-ND (http://creativecommons.org/licenses/by-nc-nd/4.0/).
\end{abstract}

\footnotetext{
* Autor para correspondencia.

Correo electrónico: i_ortiz2611@hotmail.com (I. Ortiz-Vargas).

La revisión por pares es responsabilidad de la Universidad Nacional Autónoma de México.
} 


\section{KEYWORDS}

Pressure ulcers; Home health nursing; Wound healing; Hydrocolloid bandages; Mexico

\section{PALAVRAS-CHAVE}

Úlceras por pressão; Enfermagem domiciliar; Cicatrização de feridas; Curativos hidrocoloides; México

\section{Pressure ulcers humid healing: Attention within the home environment}

Abstract The need to carry out prevention and treatment of pressure ulcers (PU) within the home environment by means of humid healing sessions is very important for the prompt eversion and cicatrization of the wounds.

Objective: To assess the efficacy of the humid healing in patients with PU within the home environment.

Method: A clinical intervention was performed using the humid healing technique on $11 \mathrm{PU}$ in 4 discharged patients showing degree II lesions $(n=6)$, and degree III lesions $(n=5)$. Twelve healings every $72 \mathrm{~h}$ were carried out at the homes of these patients. The ulcers severity and progress were estimated using the Pressure Ulcers Scale for Healing (PUSH) instrument, which has a Cronbach alfa of 0.823 .

Results: Nine PU out of the total 11 healed - all degree $॥(n=6)$ and 3 degree III $(n=5)$. The cicatrization process only took 4 weeks, a time which is much shorter than the usual 8 weeks which take the traditional healing.

Conclusion: Within the home environment, humid healing was an effective method to address PU in patients previously discharged from hospitals. This kind of interventions represents an option which health institutions can promote in order to improve the quality of life of these patients.

(c) 2017 Universidad Nacional Autónoma de México, Escuela Nacional de Enfermería y Obstetricia. This is an open access article under the CC BY-NC-ND license (http://creativecommons.org/licenses/by-nc-nd/4.0/).

\section{Cura húmida de ulceras por pressão. Atenção no âmbito domiciliar}

Resumo A necessidade de trabalhar na residência o tratamento e prevenção das Ulceras por pressão (UPP) mediante sessões baseadas em cura húmida, é transcendente para a eversão e cicatrização em menor tempo.

Objetivo: Avaliar a eficácia do tratamento da cura húmida em pacientes com UPP em um ambiente domiciliar.

Método: Realizou-se uma intervenção clínica com a técnica de cura húmida em 11 UPP de quatro pacientes formados do hospital com seu problema de saúde resolvido, mas não assim as UPP observadas em pontos de pressão, com lesões grau $(n=6)$ e $॥(n=5)$. Realizaram-se 12 curas, cada $72 \mathrm{~h}$ nas residências das pessoas. Mediu-se a severidade e sua reversão com o instrumento Pressure Ulcers Scale for Healing (PUSH). Este instrumento tem um alfa de Cronbach de 0.80 . Resultados: Foram curadas 9 UPP de 11 , todas as de grau ॥ $(n=6)$, enquanto que as de grau III, 3 $(n=5)$. A cicatrização deu-se em um lapso de 4 semanas, inferior à cura tradicional que demora duas vezes mais o tempo de cicatrização.

Conclusão: No âmbito domiciliar, a cura húmida foi eficaz para reverter as UPP de pacientes que as tinham desenvolvido durante sua hospitalização e que se formaram sem tratamento para elas. A intervenção se tornou em una opção para melhorar a qualidade de vida das pessoas e um médio que as instituições de saúde podem pôr em prática.

(c) 2017 Universidad Nacional Autónoma de México, Escuela Nacional de Enfermería y Obstetricia. Este é um artigo de acesso aberto distribuído sob a licença de Creative Commons CC BY-NC-ND (http://creativecommons.org/licenses/by-nc-nd/4.0/).

\section{Introducción}

Una úlcera por presión (UPP) es una lesión localizada en la piel $y / o$ en el tejido subyacente, por lo general sobre una prominencia ósea, como resultado de una presión ejercida y mantenida entre dos planos duros y la tolerancia de los tejidos a esta presión en combinación con el cizallamiento ${ }^{1}$. En 1958, Kosiak comprobó que una presión externa de $70 \mathrm{mmHg}$ mantenida durante $2 \mathrm{~h}$ provocaría lesiones isquémicas en todos los tejidos, puesto que la presión capilar normal de la piel oscila entre 16 y $33 \mathrm{mmHg}^{2}$.

$\mathrm{Si}$ se ejercen presiones superiores a $20 \mathrm{mmHg}$ en un área limitada y durante un tiempo prolongado, se inicia un proceso de isquemia que impide la llegada de oxígeno y nutrientes, que origina una rápida degeneración de los tejidos por la respiración anaerobia, lo que provoca alteraciones a nivel de la membrana celular, y por lo tanto causa necrosis y muerte celular ${ }^{3}$.

El signo inicial de la UPP es el eritema ocasionado por la isquemia que lesiona los tejidos. Cuando estos se destruyen, ocasiona rotura de la piel y los microorganismos inician la población de la lesión, colonizándola e infectándola.

Las UPP se presentan como lesiones redondeadas u ovaladas situadas perpendicularmente sobre las prominencias óseas del cuerpo, como talón, sacro, tobillo, codos, hombro, escápulas, todas ellas en función de la posición anató- 
mica en que la persona permanezca oprimiendo los tejidos durante tiempos prolongados ${ }^{4}$. También pueden producirse sobre tejidos blandos sometidos a presión por dispositivos de salud, como UPP en nariz por sonda nasogástrica, UPP en genitales causada por sonda Foley o sus fijadores, UPP en pabellón auricular por puntas nasales, UPP en hueso nasal por mascarillas de oxígeno, etc.

Las UPP afectan a las personas de cualquier edad que presentan poca o nula capacidad para moverse y un estado nutricional desfavorable, en especial a personas de más de 60 años y pacientes pediátricos, en áreas localizadas en zonas de apoyo coincidentes con prominencias óseas combinado con estancias hospitalarias prolongadas.

La mayoría de los personas con UPP están hospitalizadas, ingresan por patologías graves o enfermedades crónicas degenerativas en fase avanzada y sufren efectos adversos frecuentes a su estancia por permanecer inmovilizadas. Una vez que se desarrolla la UPP, se degenera la calidad de vida del paciente y se acorta su esperanza de vida, al afectar su autonomía, independencia y autoestima por las complicaciones desencadenadas, como dolor, sepsis y osteomielitis. De manera paralela se incrementan los costos sanitarios, aumentan la carga de trabajo para los profesionales de la salud, por lo cual se hace más difícil una correcta valoración y tratamiento de las UPP desarrolladas, lo que puede prolongar hasta 5 veces más su estancia hospitalaria ${ }^{5}$.

Las UPP son un riesgo innecesario para el paciente que se encuentre en cualquier latitud del planeta y uno de los efectos adversos más notorios del cuidado hospitalario de los profesionales de la salud de todo el mundo. En España, un estudio a nivel nacional reveló que el $7.8 \%$ de los pacientes con UPP eran mayores de 65 años. Los datos obtenidos proporcionaron información sobre 2,837 lesiones: $24.5 \%$ de estadio I, $42 \%$ de estadio II, $19.8 \%$ de estadio III, $13.7 \%$ de estadio iv y $0.5 \%$ sin estudiar. Los pacientes que presentaron más incidencia de UPP fueron los mayores de 65 años y aquellos en los que se daba una estancia prolongada, por arriba de 4 días $^{6}$. Un aporte importante es que el $59.4 \%$ de las UPP se originaron en el hospital ${ }^{7}$.

Precisamente por este hecho, y por la evolución del envejecimiento de la población, la atención que proporciona el cuidador principal es insustituible y la aportación de enfermería debería ser más eficiente y eficaz, ya que se abre un amplio campo de trabajo que poco ha avanzado y que invita a todos los agentes a responder a las necesidades actuales que demanda la población mundial, pues es en el ámbito de atención primaria donde la prevalencia es más alta ${ }^{8}$.

En México, un estudio informó que las entidades federativas con mayor prevalencia de pacientes que tuvieron UPP fueron: Veracruz (72.9\%), Campeche (60\%), Colima (50.7\%), Jalisco (46.1\%) y Baja California (45.8\%). Las mujeres tuvieron una mayor prevalencia de UPP (52\%), así como los pacientes con edades entre 71 y 80 años $(20 \%)^{9}$.

En cuanto a los días de aparición de las UPP, se ha reportado que el promedio fue de 6 días después de la fecha de ingreso; el $26 \%$ de los pacientes llegaron a la unidad médica con UPP ya desarrolladas y el 74\% desarrollaron UPP después de su ingreso a la unidad médica. Con relación al sitio anatómico, se encontró que la frecuencia de la aparición fue: sacro $(27 \%)$, talón $(27 \%)$, isquion $(10 \%)$, cabeza $(4 \%)$ y codos $(3 \%)^{9}$.
Se debe abrir camino en la investigación sobre el tratamiento de las UPP, ya que es uno de los indicadores adversos en el tratamiento de los pacientes según el informe de la Organización Mundial de la Salud (OMS), al cual pocos le han dado la importancia debida, pues a los pacientes se les niegan los cuidados necesarios y se prolonga el tiempo de estancia hospitalaria, por lo que se incrementan los costos de la atención ${ }^{10}$.

Cabe destacar que en el Programa Nacional de Salud se trabaja sobre la prevención de las UPP y los hospitales han implementado medidas para revertir la situación, pero no se ha alcanzado la meta deseada, pues el paciente desarrolla UPP, egresa sin tratamiento y en sus hogares permanecen aún más abandonados por los sistemas de salud, sin los medios y sin los recursos para abordar una situación tan compleja.

Las acciones antes de la aparición de una UPP deben encaminarse exclusivamente a la prevención y dirigirse específicamente a la identificación del riesgo, los cambios posturales, la higiene e hidratación de la piel, el adecuado aporte nutricional, la protección de puntos de presión y la evaluación constante de la piel ${ }^{11}$. Una vez desarrollada la UPP, las acciones tienen como prioridad abordarla inmediatamente y cicatrizar en el menor tiempo y estadio posibles.

El abordaje de las UPP empieza por identificar a los pacientes, por medio de la escala de Braden, con alto riesgo de desarrollar las úlceras, tratar de prevenirlas por medio de movilizaciones al paciente y/o mantenerlo en constante rotación con superficies especiales, como los colchones de aire alternante, además de aumentar su ingesta calórica por medio de una dieta hiperproteica e ingesta de suplementos alimenticios $^{12}$.

Es muy importante abordar las UPP con un enfoque interdisciplinario y de manera integral, pues no basta con solo curar la úlcera, sino que es necesario identificar los factores que pueden afectar o no la cicatrización de la UPP, como la nutrición del paciente, el apoyo emocional con el que cuenta, los medicamentos que tiene prescritos, el microclima que envuelve la lesión y los aspectos mismos de la herida, como tamaño, forma, tipo de tejido, cantidad de exudado, colonización bacteriana, etc.

Para el tratamiento, la técnica de cura húmeda es una terapia alternativa en contraposición a la cura seca para la eversión de la UPP. Se basa en la limpieza de la UPP por medio de desbridamientos y la utilización de apósitos que cubren la herida, con lo que se facilita el aporte de oxígeno y de nutrientes al lecho de la lesión, para favorecer la acción de los fibroblastos en la producción de colágeno y mejorar la acción bacteriostática que acidifica el pH de la zona. Todo ello conlleva ofrecer resultados en la cicatrización de la herida en un menor tiempo y, a la larga, disminuye los costos en el abordaje de la herida ${ }^{13}$.

En la técnica de cura húmeda se realiza una valoración inicial de la herida por medio de la escala Pressure Ulcers Scale for Healing (PUSH). Esta escala permite, por medio de subpuntuaciones en 3 dimensiones que se suman, conocer el grado de progresión de la UPP, donde a menor puntaje, mejor eversión de la UPP.

Las dimensiones de medición de PUSH son la longitud por anchura, si la herida está infectada o colonizada y el tipo de tejido que presenta, que puede ser necrótico, esfacelado, 
epitelizante o cerrado, además de la cantidad de exudado que va de abundante, moderado, ligero y ninguno.

Posterior a ello, se lava la herida con suero fisiológico y se cubre esta con apósitos de diferentes tipos y materiales. De acuerdo con la valoración, los apósitos pueden ser hidrocelulares, alginatos, apósitos de plata, apósitos de hidrogel, etc., cuya intención será permitir que el lecho de la herida permanezca en un ambiente húmedo para favorecer el crecimiento celular y una adecuada cicatrización ${ }^{14}$.

Además, la cura húmeda favorece el crecimiento de tejido de granulación en un ambiente adecuado, protegiéndolo de agentes externos y de agentes infecciosos. El ambiente húmedo en la herida mantiene también la temperatura adecuada para estimular la fibrinólisis ${ }^{15}$.

Por lo tanto, abordar la problemática hace necesario trabajar la curación y tratamiento en el entorno domiciliario, ya que la mayoría de los pacientes egresan de una institución con úlceras y no reciben el cuidado necesario en el hogar. El tiempo es el factor imprescindible del tratamiento de la UPP, pues mientras más rápido se trate la UPP, menor riesgo para el paciente y menor costo del tratamiento en comparación con la cura tradicional ${ }^{16,17}$.

El escenario domiciliar surge como una alternativa para el tratamiento de las UPP, ya que por motivos de distancias con relación a los centros de salud, la saturación de los mismos en los servicios de urgencias, y la lejanía de una cita con el médico para su curación, hace que los pacientes busquen alternativas poco confiables, de carencia científica y sin resultados comprobados.

Así, el objetivo fue: evaluar la eficacia del tratamiento, en un ambiente domiciliario, de la cura húmeda en pacientes con deterioro de la integridad cutánea por UPP.

\section{Método}

Se llevó a cabo una intervención clínica de tipo prospectiva y longitudinal, en el ámbito domiciliar. La muestra de la intervención fueron 4 personas egresadas de una unidad de salud con UPP y que no recibieron atención para su curación a su egreso.

La variable independiente es la cura húmeda, manejada en la intervención con 3 indicadores: desbridamiento, apósito biocompatible y dolor. La variable dependiente es la UPP, la cual tiene 7 indicadores: localización, grado, aspecto, longitud $\times$ anchura, cantidad de exudado y tipo de tejido. La unidad de análisis es la reversión de las UPP en los pacientes.

Criterios de inclusión: haber egresado de la unidad hospitalaria, mayor de edad, sin importar el sexo, tener al menos una UPP, independiente de las condiciones de esta y los tratamientos que usaron para tratarla, que aceptaron participar voluntariamente en el estudio.

Criterios de exclusión: los pacientes que presentaron una úlcera que no fuera por presión.

Se aplicó el instrumento PUSH, el cual se traduce como «Escala para la curación de la úlcera por presión». Fue desarrollado por el National Pressure Ulcer Advisory Panel (NPUAP), como una herramienta rápida y confiable para monitorear el cambio en el estado de una UPP en el tiempo, adoptado como Documento GNEAUPP (Grupo Nacional para el Estudio y Asesoramiento de las Úlceras Por Presión y heridas crónicas), previa autorización del NPUAP ${ }^{18}$.

Este instrumento permite documentar la cicatrización de una UPP por medio de una medición al observar la úlcera. Su alfa de Cronbach fue $\geq 0.80$ en estudios previos.

Se observa la UPP, se mide y se clasifica con respecto a 3 categorías: longitud, cantidad de exudado y tipo de tejido de la herida.

La longitud por anchura es un subvalor que depende del resultado de la medición en $\mathrm{cm}^{2}$ (donde 0 es $0 \mathrm{~cm}^{2}, 1$ es $<.3 \mathrm{~cm}^{2}, 2$ oscila entre $.3 \mathrm{y} .6 \mathrm{~cm}^{2}, 3$ oscila entre $<.7 \mathrm{~cm}^{2}$ y $1 \mathrm{~cm}^{2}, 4$ oscila entre 1.1 y $2.0 \mathrm{~cm}^{2}, 5$ oscila entre $2.1 \mathrm{y}$ $3 \mathrm{~cm}^{2}, 6$ oscila entre 3 y $4.0 \mathrm{~cm}^{2}, 7$ oscila entre 4.1 y $8 \mathrm{~cm}^{2}$, 8 oscila entre 8.1 y $12 \mathrm{~cm}^{2}, 9$ oscila entre 12.1 y $24 \mathrm{~cm}^{2}$, y 10 es $\left.>24 \mathrm{~cm}^{2}\right)^{19}$.

La cantidad de exudado se estima al retirar el apósito, y antes de realizar la curación por cura húmeda se observa la cantidad en el interior de la UPP ( 0 es ninguno, 1 significa ligero, 2 es moderado y 3 abundante) y el tipo de tejido de la herida ( 0 significa tejido cerrado, 1 significa tejido epitelizado, 2 significa tejido de granulación, 3 significa tejido esfacelar y 4 significa tejido necrótico). Para obtener la puntuación total, se suman los marcadores anteriores. Una comparación de las puntuaciones totales medidas en el tiempo proporciona una indicación de la mejora o del deterioro en la cicatrización de la UPP ${ }^{7,19}$.

Ex profeso, se diseñó una bitácora de observación respecto a la reversión de la úlcera, la cual recoge los datos del paciente y la descripción de la úlcera en estudio, además de incluir los datos más relevantes, como estadio, dolor y tipo de desbridamiento. Por otra parte, recoge las puntuaciones finales de la escala PUSH en forma de gráfica desde la valoración inicial, que es la sesión uno, hasta la sesión 12, donde concluye la intervención. Las mediciones van del 0 al 17, donde a menor número, mayor eversión de la UPP. La medición de la UPP se realiza en cada curación de la misma, donde, a lo largo de las curaciones, se forma una gráfica que debe ir en forma descendente para comprobar la eversión de la UPP.

Se realizó una visita previa a la intervención a todos los pacientes, en la cual se describió la intervención y una vez que accedieron se establecieron las sesiones y los horarios de común acuerdo.

En todas las sesiones se utilizó una bitácora de observación para la reversión de la úlcera, creada con la finalidad de mejorar el control y la evolución de las úlceras durante la intervención de cada uno de los pacientes. El registro se realizó por úlcera. La bitácora incluyó los datos referentes al paciente en la parte superior izquierda, el instrumento a utilizar en la parte superior derecha y las calificaciones dadas por cada variable. En el centro de la misma, la puntuación PUSH, a modo de gráfica, y en la parte inferior las características de la úlcera, como dolor, tipo de desbridamiento, el aspecto de la úlcera y el estadio.

De la misma forma, los desbridamientos en todos los pacientes fueron de tipo autolítico y de tipo cortante en las situaciones que lo ameritaban. El desbridamiento autolítico es el método más selectivo, atraumático e indoloro. El material utilizado en las curaciones fueron guantes estériles, solución salina fisiológica y gasas estériles. Se limpiaron las lesiones en cada cura con una jeringa de $20 \mathrm{cc}$ y una aguja de $19 \mathrm{~mm}$. Se cargó la jeringa de solución salina y se 
realizó el lavado para arrastrar tejidos necróticos, esfacelos y detritus celulares fuera de la úlcera.

Posteriormente, se cubrió con el apósito necesario para el tratamiento de cada paciente. Se siguió el mismo procedimiento en todas las curaciones. El desbridamiento cortante se realizó a pie de cama. El material utilizado en el procedimiento fueron guantes estériles, solución salina fisiológica, jeringa de $20 \mathrm{cc}$, una aguja de $19 \mathrm{~mm}$ y una hoja de bisturí número 15. La forma de realizarlo fue bajo técnica aséptica, se desbridó todo el tejido no viable de la herida, y se limpió con gasa estéril el excedente de exudado. Después se realizó limpieza con suero fisiológico y se cubrió con apósitos de cura húmeda.

Se planearon 12 sesiones para la intervención. Cada una de ellas con una duración de $50 \mathrm{~min} 3$ veces a la semana durante un periodo de 4 semanas. Cabe mencionar que el modo de la intervención consistió en sesiones individualizadas.

Las sesiones se integraron con una introducción de $5 \mathrm{~min}$ en la cual se preparaba el material a utilizar. Posterior a ella, se procedía a la valoración del paciente y su curación en un lapso de $35 \mathrm{~min}$, donde se revisaba la úlcera, se medía su evolución, se comparaba con las sesiones anteriores y se realizaba la curación con la técnica de cura húmeda.

La cura húmeda favorece el crecimiento de tejido de granulación en un ambiente adecuado, protegiéndolo de agentes externos y de agentes infecciosos. En la técnica de cura húmeda se lavó la herida con $60 \mathrm{cc}$ de suero fisiológico a una temperatura de $37^{\circ} \mathrm{C}$ con una jeringa de $20 \mathrm{cc}$ y una aguja $18 \mathrm{G} \times 11 / 2(1.20 \times 40 \mathrm{~mm})$.

Se secó el excedente de la solución con una gasa estéril y se utilizaron apósitos hidrocoloides y de poliuretano, para que el lecho de la herida permaneciera en un ambiente favorecedor del crecimiento celular y que permitiera una adecuada cicatrización. Los últimos 5 min eran la conclusión y despedida, al mismo tiempo que se señalaban las indicaciones pertinentes.

Para el análisis estadístico, los resultados obtenidos en las bitácoras se capturaron en Excel y se creó una base de datos con el programa SPSS. Se realizó un análisis exploratorio de las variables, así como entre las medias, la prueba de normalidad Kolmogorov-Smirnov y, por último, la estadística inferencial con la prueba de rangos de Wilcoxon. Se utilizó para su análisis estadístico el programa SPSS en su versión 21 para Windows y para la realización de gráficos se empleó el programa Statistica 7.

El protocolo de la intervención fue revisado y aprobado por el Comité de Ética y de Investigación de la Facultad de Enfermería, Región Veracruz de la Universidad Veracruzana. Cada paciente estuvo en libertad de abandonar el estudio en cualquier momento. La intervención contempló un riesgo mínimo para las personas que participaron en ella, por ser un estudio prospectivo que emplea procedimientos profilácticos no invasores, según el Reglamento de la Ley General de Salud en Materia de Investigación para la Salud, art. 17.

Los métodos utilizados no involucraron experimentos clínicos. Los participantes fueron invitados y accedieron de manera voluntaria a colaborar con el estudio, facilitando su consentimiento informado de forma verbal y por escrito. Al mismo tiempo, el responsable de realizar la intervención preservó en todo momento la confidencialidad de los datos mediante la codificación de los nombres de los pacien-

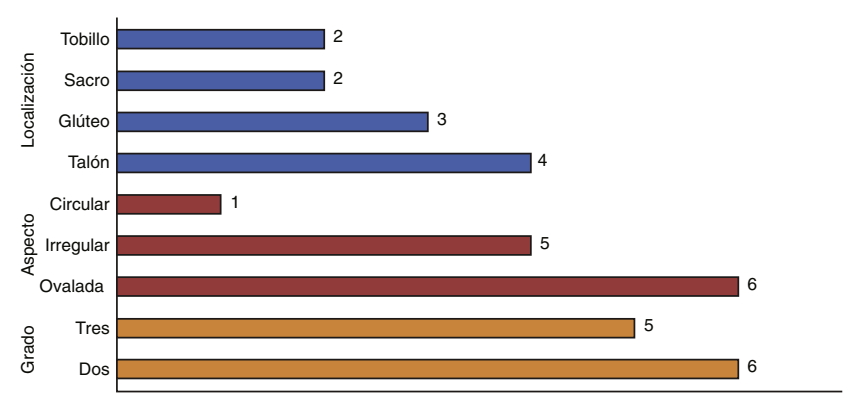

Figura 1 Localización, aspecto y grado de las úlceras por presión.

tes. De acuerdo con el reglamento de la Ley General de Salud en Materia de Investigación para la Salud, en base al título segundo, capítulo I, esta intervención cumplió con los siguientes artículos: 13, 16, 20 y 21 .

Según la Declaración de Helsinki, promulgada por la Asociación Médica Mundial (WMA, por sus siglas en inglés) en 1964, la intervención tuvo como eje el respeto por el individuo (artículo 8), su derecho a la autodeterminación y el derecho a tomar decisiones informadas (artículos 20, 21 y 22 ), incluso la participación en la investigación, tanto al inicio como durante el curso de la investigación.

Según el Código de Ética para las enfermeras y los enfermeros en México, se cumplió en la intervención con los principios de beneficencia y no maleficencia, entendidos como la obligación de hacer el bien y evitar el mal y el principio de autonomía, que significa respetar a las personas como individuos libres y tener en cuenta sus decisiones, producto de sus valores y convicciones personales.

\section{Resultados}

El instrumento utilizado para la intervención tuvo un alfa de Cronbach de 0.823 en este trabajo. Durante la investigación, los 4 pacientes presentaron úlceras con similares características. Se atendieron 6 UPP con grado $\|$ y 5 con grado II. Con relación al aspecto de las UPP, todas se formaron sobre prominencias óseas. De acuerdo con la localización, se deduce que todos los pacientes adquirieron sus úlceras por falta de movilidad durante su estancia hospitalaria, pues la postura en que permanecieron fue en semifowler y/o decúbito supino, ya que las úlceras en su mayoría se encontraban en los puntos de presión mencionados anteriormente (fig. 1).

El paciente número 1 tuvo 2 UPP, una en cada tobillo. La úlcera del tobillo izquierdo fue de grado III, tuvo una reversión lenta: en puntaje PUSH inició en 16 y terminó en 12 . La úlcera localizada en el talón derecho fue de grado॥, cedió hasta la semana 11, inició con puntaje PUSH de 13 y terminó en 0 .

El paciente número 2 tuvo 3 UPP, 2 de grado II localizadas en ambos talones y una en sacro de grado॥. La del talón derecho inició en escala PUSH de 16 y la del talón izquierdo en 15; ambas terminaron en 0 hacia la semana 12. La úlcera en sacro pudo curarse en 10 sesiones; inició con puntaje de 12.

El paciente número 3 presentó 2 UPP, una en el glúteo y otra en el sacro; ambas UPP eran de grado I. La UPP localizada en el glúteo inició con puntaje PUSH de 10; en la 


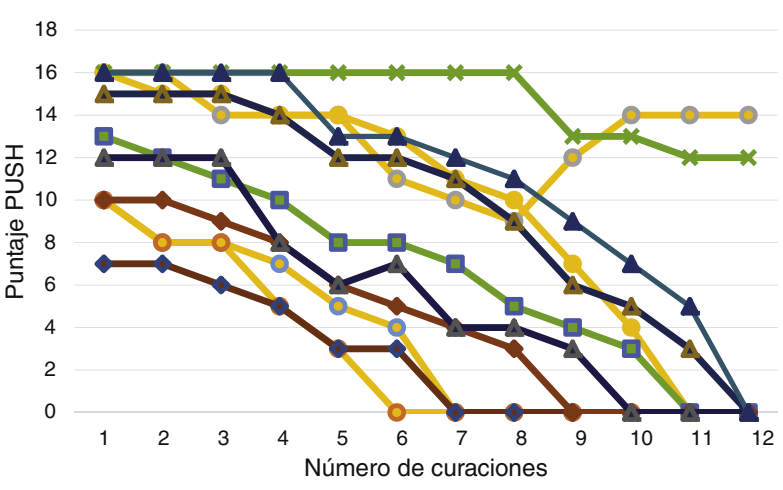

- Glúteo izq. Pac. 4 - - Glúteos der. Pac. $4=$ Talón izq. Pac. 4 Talón der. Pac. 4 -Tobillo izq. Pac. 1 -Tobillo der. Pac. 1 Glúteo der. Pac. Sacro Pac 2 -Talón izq. Pac $2 \approx$-Talón der. Pac. 2

Figura 2 Puntaje Pressure Ulcers Scale for Healing (PUSH) por úlcera durante las 12 sesiones $(n=11)$.

curación 9 la UPP ya medía 0 . Con respecto a la UPP del sacro, inició con un puntaje PUSH de 7 y en la semana 7 medía 0.

El paciente número 4 presentó 4 UPP, 2 de grado॥ en ambos glúteos y 2 de grado II en ambos talones. Las lesiones en los glúteos tuvieron un comportamiento casi idéntico: iniciaron en PUSH de 10 y terminaron en 0 hacia la semana 6 el glúteo derecho y hacia la semana 7 el glúteo izquierdo. Las lesiones en los talones iniciaron con puntaje PUSH de $16 \mathrm{y}$ solo el talón derecho se curó en la semana 11, mientras que la úlcera en el talón izquierdo hacia la sesión 12 puntuó 14 (fig. 2).

También, se midió la diferencia de medias del total de úlceras al inicio de la intervención y al final, con el fin de observar el avance de las úlceras en las áreas que abarca el instrumento PUSH. El puntaje final PUSH inició con una $\bar{x}$ de 78 para terminar en una $\bar{x}$ de 15 . Con relación al índice de longitud por anchura, inició con una $\bar{x}$ de 80 y terminó con una $\bar{x}$ de 17 (fig. 3). Además, existió significación estadística con relación al antes y después de cada una de las variables del instrumento PUSH bajo el estadístico de rangos de Wilcoxon (tabla 1).

\section{Discusión}

La técnica de cura húmeda para tratar las UPP demostró ser un tratamiento efectivo a nivel domiciliario en 9 de las 11 úlceras en un lapso de 4 semanas, preferente a la cura tradicional, la cual ocupa más del doble de tiempo para mostrar efectividad, según Soares Pott et al. ${ }^{20}$. A nivel domiciliario la presente intervención obtuvo un mejor porcentaje con relación al estudio de García Fernández et al. ${ }^{21}$, el cual mostró una eficacia del $63.5 \%$ bajo la técnica de la cura húmeda en contraposición al $81.8 \%$ en esta intervención.

Los aspectos que influyeron en estos resultados favorables fueron que, en el presente estudio, las UPP que se curaron no tenían signos de infección, las curaciones se realizaron conforme a lo planeado y se abordaron en menos de $72 \mathrm{~h}$ de haber egresado del hospital, mientras que en las 2 que no se curaron, hablamos de falta de apego al tratamiento por parte del paciente.

Se obtuvieron resultados favorables en la disminución de úlceras grados $\|$ y III, similares a los de García Fernández

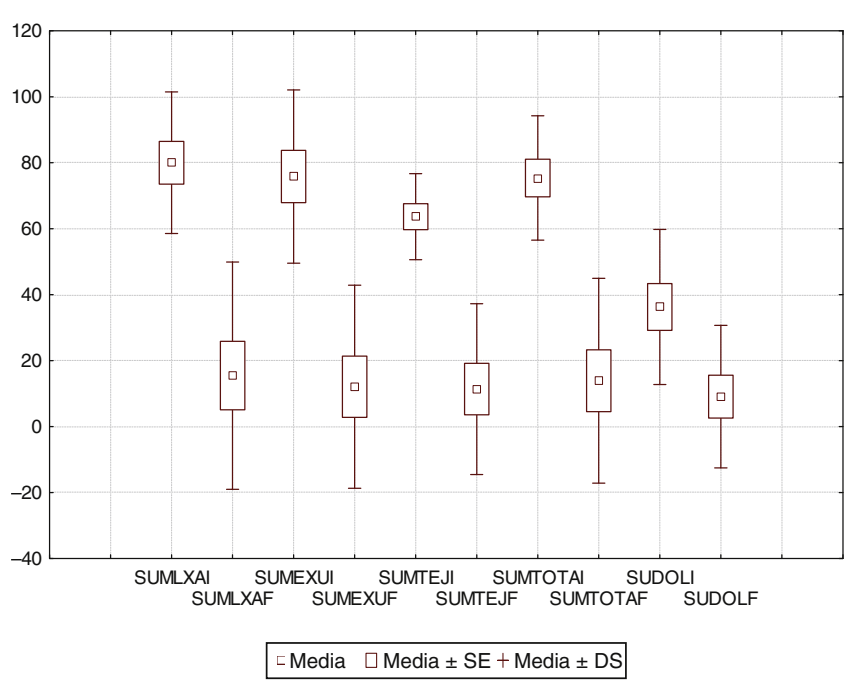

Figura 3 Comparación de los porcentajes inicial y final de las úlceras por presión $(\mathrm{n}=11)$.

SUDOLF: sumatoria dolor final; SUDOLI: sumatoria dolor inicial; SUMEXUF: exudado final; SUMEXUI: exudado inicial; SUML$X A F$ : longitud $\times$ anchura final; SUMLXAI: longitud $\times$ anchura inicial; SUMTEJF: tipo de tejido final; SUMTEJI: tipo de tejido inicial; SUMTOTF: puntaje PUSH final; SUMTOTI: puntaje PUSH inicial.

et al. ${ }^{21}$ bajo la técnica de cura en ambiente húmedo, por lo cual se mejoró la síntesis de colágeno, la proliferación celular y, por ende, la cicatrización de la herida.

Con relación a la localización de la úlcera, se observan cifras similares a las dadas por Vela-Anaya', quien mencionó que la frecuencia del sitio anatómico de las UPP fue el sacro en un $27 \%$ y el talón en otro $27 \%$. Pesantes Masabanda ${ }^{22}$ menciona en su investigación la localización de las úlceras en sacro, talones y glúteos, pues son las mayores prominencias óseas que cizalla la piel en la posición anatómica decúbito supino con menor cantidad de tejido.

La poca movilidad de las personas en los hospitales, la sobrecarga de trabajo entre los profesionales de la salud y el desconocimiento de los familiares ocasionan una mayor probabilidad de desarrollar UPP en un corto tiempo de estancia.

La cura húmeda con los diferentes tipos de apósitos que se utilizan, como lo destacan Clark et al. ${ }^{23}$, no es comparable con los alginatos, hidrogeles, poliuretanos, etc., ya que en esta investigación solo se usan hidrocoloides. En términos de eficacia, la cicatrización de la herida no solo depende de la cura húmeda o del tipo de apósito, sino que alrededor de la técnica hay otros factores, como la edad del paciente, el aumento del soporte nutricional y/o la satisfacción de sus necesidades psicosociales, además de la complejidad del proceso de cicatrización ${ }^{11,24,25}$.

\section{Conclusión}

La investigación demostró la eficacia del tratamiento de la cura húmeda en pacientes con deterioro de la integridad cutánea por UPP en un ambiente domiciliario.

La técnica en ambiente húmedo mejoró el tiempo de cicatrización de las UPP en comparación con la técnica tradicional de cura en ambiente seco. 
Tabla 1 Estadístico de contrastes Wilcoxon $(n=11)$

\begin{tabular}{|c|c|c|c|c|c|}
\hline & $\begin{array}{l}\text { Longitud por altura } \\
\text { inicial vs. final }\end{array}$ & $\begin{array}{l}\text { Exudado inicial vs. } \\
\text { final }\end{array}$ & $\begin{array}{l}\text { Tipo de tejido } \\
\text { inicial vs. final }\end{array}$ & $\begin{array}{l}\text { Puntaje PUSH } \\
\text { inicial vs. final }\end{array}$ & $\begin{array}{l}\text { Sumatoria dolor } \\
\text { inicial vs. final }\end{array}$ \\
\hline Z & $-2.944 b$ & $-2.850 b$ & $-2.859 b$ & $-2.941 b$ & $-2.828 b$ \\
\hline Sig. asintót. (bilateral) & 0.003 & 0.004 & 0.004 & 0.003 & 0.005 \\
\hline
\end{tabular}

Para tratar todas las UPP, se debe hacer una valoración integral que incluya factores de riesgo, escala de Braden, revisión de la piel, el tipo de tejido, cantidad de exudado, piel perilesional, aspecto de la úlcera y localización, además de los factores intrínsecos de la persona, como la nutrición y el soporte emocional.

Esta investigación invita a un cambio de cura en los ambientes hospitalarios y profesiones; aun cuando el tiempo y los insumos son insuficientes, es necesaria una modificación del abordaje por el coste-beneficio del paciente y de la institución.

Se necesita de investigaciones con más población de estudio para la confirmación de la eficacia de la técnica de la cura húmeda.

La intervención domiciliaria supone beneficios para el paciente que padece UPP, y que muy probablemente presenta un alto riesgo de tener dificultad para moverse, disminuye los costos para el familiar, al mismo tiempo que mejora la calidad de curación al no estar el profesional comprometido a hacer tantas curaciones en una jornada de trabajo. También se podría otorgar educación al familiar y al paciente sobre prevención, cuidado y tratamiento. La intervención resulta individualizada, más cercana y cálida, al estar en un entorno que el paciente considera seguro como su hogar.

\section{Responsabilidades éticas}

Protección de personas y animales. Los autores declaran que los procedimientos seguidos se ajustaron a las normas éticas del comité de experimentación humana responsable y de acuerdo con la Asociación Médica Mundial y la Declaración de Helsinki.

Confidencialidad de los datos. Los autores declaran que han seguido los protocolos de su centro de trabajo sobre la publicación de datos de pacientes.

Derecho a la privacidad y consentimiento informado. Los autores han obtenido el consentimiento informado de los pacientes y/o sujetos referidos en el artículo. Este documento obra en poder del autor de correspondencia.

\section{Financiamiento}

Ninguno.

\section{Conflicto de intereses}

Los autores declaran no tener conflicto de intereses.

\section{Referencias}

1. Restrepo-Medrano JC, Escobar CL, Cadavid LM, et al. Propuesta de atención al paciente con úlceras por presión (UPP) a través del proceso de atención de enfermería (PAE). Medicina U.P.B. 2013;32:68-78.

2. Blanco López JL. Definición y clasificación de las úlceras por presión. Peu. 2003;23:194-8.

3. Soldevilla-Agreda JJ. Las úlceras por presión en Gerontología. Dimensión epidemiológica, económica, ética y legal. Santiago de Compostela: Universidad de Santiago; 2007.

4. Montoya-Carralero MD, Ríos-Díaz J, Martínez-Fuentes J. Prevención de las úlceras por presión en pacientes terminales e inmovilizados seguidos por el equipo de soporte de atención domiciliaria. Rev Esp Geriatr Gerontol. 2007;42:263-70, http://dx.doi.org/10.1016/S0211-139X(07)73561-8

5. Smeltzer SC, Bare BG, Hinkle JL, et al. Enfermería médicoquirúrgica. 12. ${ }^{\mathrm{a}}$ ed. New York: Lippincott; 2013.

6. Soldevilla-Agreda JJ, Torra i Bou JE, Verdú Soriano J, et al. Tercer Estudio Nacional de Prevalencia de Úlceras por Presión en España, 2009: epidemiología y variables definitorias de las lesiones y pacientes. Gerókomos. 2011;22:77-90.

7. Tomé Ortega MJ. Las úlceras por presión como efecto adverso de la hospitalización. Enferm Dermatol. 2010;4:35-41.

8. Ponce-Martínez DE, Martínez-López R. Valoración de los conocimientos del cuidador principal sobre úlceras de presión. Enferm Glob. 2011;10:60-84.

9. Vela-Anaya G. Magnitud del evento adverso: úlceras por presión. Rev Enferm Ins Mex Seguro Soc. 2013;21:3-8.

10. Organización Mundial de la Salud. Día Mundial de la Salud 2012 ¿Está usted preparado? Ginebra: OMS; 2012.

11. Valero-Cárdenas H, Parra DI, Rey Gómez R, et al. Prevalencia de úlcera por presión en una institución de salud. Rev Univ Ind Santander. 2011;43:249-55.

12. Organización Panamericana de la Salud (OPS). Úlceras por presión: Guía de diagnóstico y manejo. Parte II. México: OPS, s/f.

13. Arantón-Areosa L, Delgado-Fernández R, Calvo-Pérez Al, et al. Diseño e interpretación del catálogo de productos de cura en ambiente húmedo del SERGAS. Enferm Dermatol. 2016;10: $12-8$.

14. Gonzales-Santamaría ES, Palacios-Arbeláez LT, Ruiz-Cataño M. Clínica de heridas en la ciudad de Medellín [Tesis]. Medellín: Universidad CES; 2011

15. Grupo de trabajo de úlceras por presión (UPP) de La Rioja. Guía para la prevención, diagnóstico y tratamiento de las úlceras por presión. Logroño: Consejería de Salud de La Rioja; 2009.

16. Rocha AP, Rodrigues A, Silva AJ, et al. Metodologías educativas para la prevención de las úlceras por presión: estudio piloto en las islas Azores. Gerókomos. 2014;25:41-3, http://dx.doi.org/10.4321/S1134-928X2014000100009

17. Fernández RD, Borrego Caro MJ, Díaz Rey MJ. Panel de recomendaciones sobre la combinación de apósitos para la cura en ambiente húmedo. Enferm Dermatol. 2014;8:15-21.

18. González-Consuegra RV, Verdú-Soriano J. Calidad de vida y cicatrización en pacientes con úlceras de etiología venosa. Validación del Charing Cross Venous 
Ulcer Questionnaire, versión española (CCVUQ-e) y del Pressure Ulcer Scale for Healing, versión española (PUSHe). Resultados preliminares. Gerokomos. 2011;22:131-6, http://dx.doi.org/10.4321/S1134-928X2011000300008

19. GNEAUPP. Instrumento para la monitorización de la evolución de una úlcera por presión. Logroño, España; 2015.

20. Soares Pott F, Meier MJ, Dorociak Stocco JG, et al. La efectividad del hidrocoloide versus otros apósitos en la cicatrización de úlceras por presión en adultos y ancianos: revisión sistemática y metanálisis. Rev Latino-Am Enfermagem. 2014;22:511-20, http://dx.doi.org/10.1590/0104-1169.3480.2445

21. García Fernández FP, Pancorbo Hidalgo PL, Verdú Soriano J, et al. Eficacia de los productos para el tratamiento de las úlceras por presión: una revisión sistemática con metanálisis. Gerókomos. 2007; 18:36-48.
22. Pesantes Masabanda VE. Informe de investigación sobre: Intervención de enfermería en pacientes con úlceras por presión asociados a factores de riesgo en pacientes hospitalizados en el servicio de medicina interna [Tesis]. Ecuador: Universidad Técnica de Ambato; 2012.

23. Clark M, Black J, Alves P, et al. Systematic review of the use of prophylactic dressings in the prevention of pressure ulcers. Int Wound J. 2014;11:460-71.

24. Jull $A B$, Cullum N, Dumville JC, et al. Honey as a topical treatment for wounds. Cochrane Database Syst Rev. 2015;6, http://dx.doi.org/10.1002/14651858.CD005083.pub4

25. Benoit R, Mion L. Risk factors for pressure ulcer development in critically ill patients: A conceptual model to guide research. Res Nurs Health. 2012;35:340-62. 